\title{
Automotive Health Monitoring System
}

\author{
Simran Arora, Surbhi Sen, Sandeep Singh Rawat, Swati Arora \\ Department of Electronics and Communication Engineering, Swami Keshvanand Institute of Technology, \\ Management \& Gramothan, Jaipur, Rajasthan (INDIA) \\ Email: arora.simran1702@gmail.com, surbhitbm@gmail.com,Sandeeprawat7800677632@gmail.com, \\ aroraswati14@gmail.com \\ Received 09.02.2021, received in revised form 18.03.2021, accepted 04.04.2021
}

\begin{abstract}
In today's era we see almost all the families around us are owners of an automobile, and with the fact that any machine needs a proper maintenance strategy we have observed the strategies being used for the automobiles. Maintenance strategy being used in automotive industry is normally a reaction to mishap to the vehicle that results in reduction of lifetime of vehicle and also loss of money in life. Almost maximum families use cars as their primary choice of transportation, but not all can afford higher end cars with world class facilities, so we aim to make a device that continuously detects the functioning of the cars and provide details of it to the owners on their mobile application (at an affordable price). This will not only detect the conditions of the car but also highlight the parts that require maintenance and ensure safety of both the car and its owner. The details of the car will be provided in the efficient and effective manner with the help of data and bar graphs. At this stage we feel the need of proactive maintenance to keep the details of the car up to date with us. Cars needs effective and strategical maintenance as they have complex structure so to improve their performance their servicing needs to be done smartly and efficiently. Now our work of proactive maintenance uses the interfacing of the hardware and software using sensors and android application to give a daily report of the vehicle to its owner so that they can take care of their vehicles according to it. It helps in cost cutting and as well as hassle free maintenance.
\end{abstract}

Keywords-Maintenance strategy, automobile, mobile appli- cation, bar graphs, strategical maintenance

\section{INTRODUCTION}

As an automotive became a major part of our society and as their hardware and software are both complex in nature and hence their maintenance is also a challenged for us. So, it means a proper maintenance to prevent the automotive from future wear and tear. To prevent that wear and tear we need a proactive maintenance that includes a detail of automotive and provides to its owner so that they can take care of their automotive proactively. It act as a tracker for the automotive so that it sends selfexamine itself and find what cause in the problem and gives the data to automotive owner. It leads in cost cutting and hassle free maintenance that saves the time. While in periodic servicing a vehicle is being schedule for a period of time of the distance covered by the vehicle but what happened is the vehicle is out of order in that period of time. That where our automotive health monitoring system come to its action. In the process of making our system in action that data is being collected from different sensors that are installed in different part of our vehicle. And then the data that collected from the sensors is being sent over the internet backend application for analytical and decision-making purpose. The data being collected shows the condition of the vehicle, whether it has some problem in it or not. If some part shows that they need immediate attention or not it will be taken care of. While making our system in action result it saving a lot of money in terms of servicing and maintenance charges. That makes the vehicle in perfect shape and it helps the need of attention that only the defective parts of the vehicle needed. The check includes the pressure measurement, engine oil flow rate, or fuel temperature and pressure.

\section{PERIODIC MAINTENANCE}

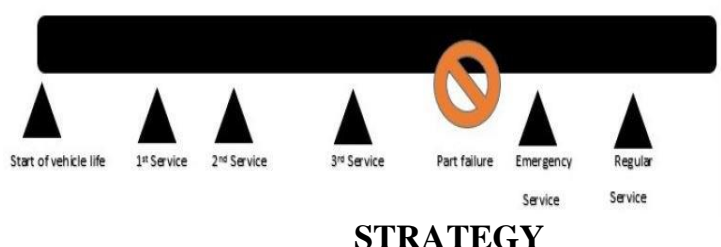

Fig.1. General Periodic Approach

Periodic maintenance strategy involves those activities which are performed on equipment's based on a set of time interval. Generally periodic car maintenance is scheduled within 6 months of last service date or after travelling 10,000 kilometers whatever happens first. The main motive of this strategy is to maintain smooth performance of the car. The assumption that the failure of assets can be predicted by time is the efficacious of the periodic maintenance strategy.

\subsection{Working of Periodic Maintenance}

There are various steps in working of periodic maintenance:

In periodic maintenance the first step, is to specify an asset.

- Then analyze the historical data which is used in main- tenance.

After analyzing historical data, set the periodic 
mainte- nance time period.

- Then normally run the assets.

- Further receive the periodic maintenance reminder.

- And at last perform the periodic maintenance.

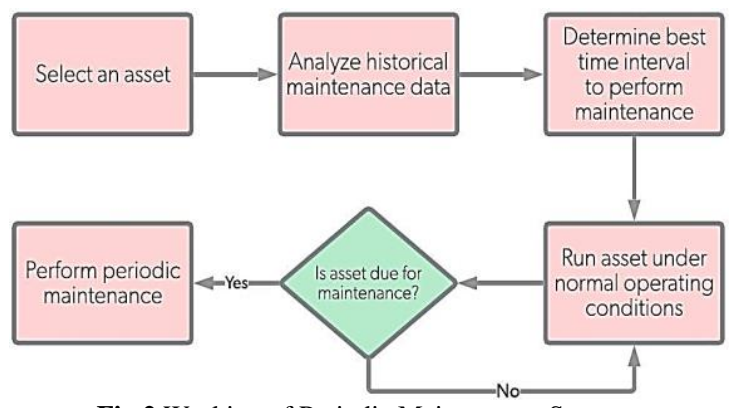

Fig.2 Working of Periodic Maintenance Strategy

\subsection{Disadvantages of periodic maintenance}

Periodic maintenance strategy has a lot of demerits asso- ciated with it, few of them can be listed as, if some parts of the vehicle malfunctions and needs immediate attention but will not come to be known in this strategy until and unless that part completely breaks down, thus we can say this strategy is dependent on historical data and analysis so the additional physical device or servicing is costly as well as it will be a reactive approach of maintenance. Keeping the hazardous effects this approach can have to the life of the person inside the car, a new approach, that is, a proactive maintenance approach or a predictive maintenance strategy can be adopted wherein the data from different sensors installed in the vehicle, related to the health status of the different parts of the vehicle and send it over the internet to backend application for analytical and decision-making purposes.

\subsection{Benefit of time-based maintenance}

The merits of having periodic car maintenance include the fact that it does not require any extra appliances to decide when appliances need to be serviced. Regular changing of oil, coolants, radiator fluid, and other vital fluids vastly improve fuel efficiency. In it complete servicing is done, whereas now only the part which need attention will be serviced.

\section{PREDICTIVE MAINTENANCE STRATEGY}

Predictive maintenance strategy or proactive strategy is concerned with the technology in which study of a particular component is done with the help of sensors to predict when that equipment might fail so that maintenance can be done just before that happens.

In this approach sensors provide real time data of the equipment which is then used to predict and prevent equipment failure.

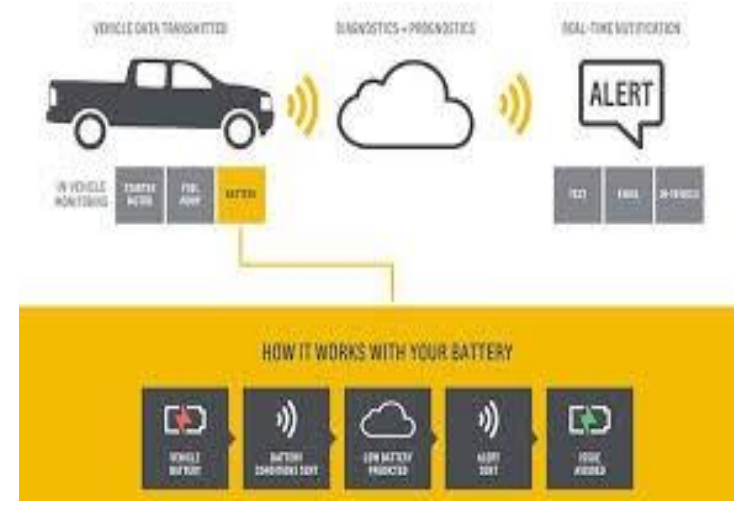

Fig. 3. General Predictive Approach

\section{A. Working of Predictive Maintenance}

Predictive maintenance strategy uses real-time data and historical data from different sensors installed in the vehicle, related to the health status of the different parts of the vehicle and send it over the internet to back-end application for analytical and decision-making purposes. There are various steps in working of predictive maintenance:

- The first step of predictive maintenance is to set up baseline.

Second step is to install conditional monitoring system, So, that we need to monitor assets conditional baseline and collect the data before installing sensors.

In this step, the collected data is compared to control any abnormalities.

- In last step, we analyze the problem in the vehicle. And with the help of the analytical application we provide a work flow and schedule an appointment with the service provider, if some parts of the vehicle need an immediate attention.

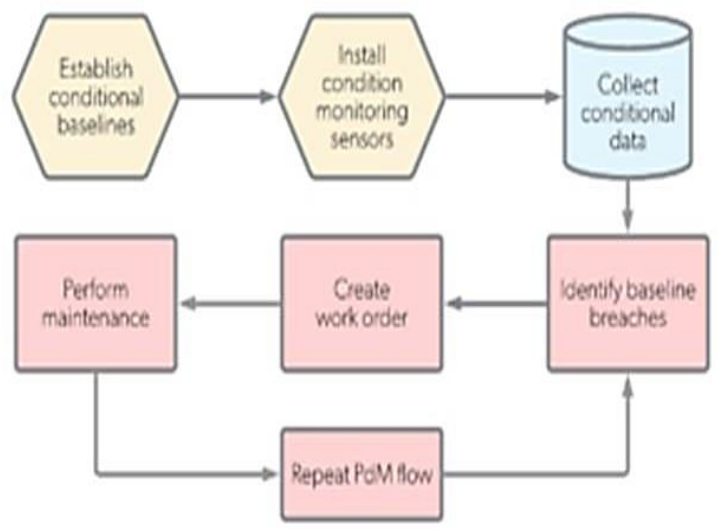

Fig. 4. Working of Predictive Maintenance Strategy

\section{B. Benefit of Predictive Maintenance}

Predictive maintenance strategy study has shown the following beneficiary results: Reduction in maintenance cost by 25-30 percent, decrease in breakdowns by $70-75$ percent, 10times return on investments, 20-25 percent increase in production and reduction in downtime by 35-45 percent.

It also benefits in the following aspects:

- It minimizes the time the equipment is maintained 
and the cost of spare parts and supplies.

- It minimizes the production hours lost to maintenance.

- It improves safety.

\section{LITERATURE REVIEW AND FINDINGS}

We have observed that in nowadays predictive maintenance approach is playing a vital role in automotive vehicle in- dustry. Previously it was hard to diagnose specific designing exertions because of the limited availability of sensors. At this time there is a great progress in automotive vehicle industry, with the help of machine learning techniques for failure prediction it has become possible to examine sensor's data. The revolution in technologies nowadays seems to be endless and in automotive system the modern cars is advanced towards integration of microprocessors, sensors etc. Nowadays there is various number of microprocessors and sensors is fitted in cars therefore the cars are more smarter in terms of engine decisions, performance, fuel efficiency, security and stability. Machine learning methods, soft computing, on-board data analysis and sequential pattern mining algorithm these recent technologies being used in automotive vehicle industry. The key area of interest in present situation is On-Board Diagnostics (OBD). Reporting capability and self-diagnostic is an automotive tenure mentioning in vehicle's. The status of the different vehicle sub-systems provides to the vehicle owner or repair technician in On-Board Diagnostics system. The different shapes of On-Board Diagnostics system are: OBD-1:- For the vehicle's useful life the controlling intent of OBD-1 was to inspire the auto manufacturers to proposed areliable emission control systems.

OBD-1.5:- In OBD 1.5 there is an additional vehicle-certain diagnostic and control circuits are available. It is signifies the limited implementation of OBD-II which is general motors used on several vehicles in 1994,1995 and 1996.

OBD-2:- In capability and standardization there is a devel- opment in OBD-II compare to OBD-I. In OBD-II there is a specification in the type of diagnostic connector and its pin out, the electrical signaling protocols available, and the messaging format. It is monitor the issues of vehicle alongside how to encode the data is available in candidate list.

As a outcome of this standardization, a particular device can enquire the on-board computer in several vehicle. GPS, AC sensors, fuel sensor, rear camera, temperature sensor, inertial sensor, exhaust gas sensor, pressure sensor, infrared sensor, water flow sensor, RADAR sensor, clutch plates sensors, video cameras, etc. these sensors are operated for functionality checking. The major intent of this paper is to study the data from the sensors and process the data on an android application or a website to observe the vehicle health condition in real time.

Now coming to the sensors that could be used for checking the functionality of the car include GPS, AC sensors, rear camera, fuel sensor, temperature sensor, exhaust gas sensor, inertial sensor, pressure sensor, infrared sensor, RADAR sensor, water flow sensor, clutch plates sensors, video cameras, etc.

The main objective of this paper is to explore the data from the sensors and compute the data on an android application or a website to monitor the vehicle health status in real time.

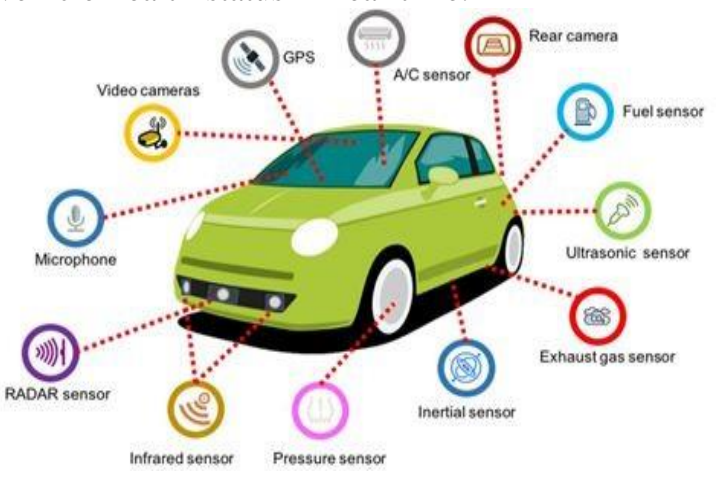

Fig. 5. Data to be diagnosed from cars

\section{PROPOSED SYSTEM}

\subsection{Software}

- The first step includes understanding the concept for which we need to generate a code and frame a solution.

- Then divide the whole concept in different modules and design a flow chart and pseudo codes for each module, and finally write the code for the modules using arduino codes.

- Compile the code and check for errors, if any do the necessary troubleshooting.

- Make the hex files of the program using avrstdio and finally Burn the program into the controller using preload burner.

With the help of GSM technology the real time data recorded with the help of various sensors installed in an automotive system can be transmitted to the cloud services and can be stored in a database.[4] The data now available at the cloud platform can be presented in various forms like bar graphs, pie charts, etc. so that it becomes effective for the user to understand the reading's and get the day to day conditions of the functioning parts of the car. Also with the help of Machine Learning (ML) concept we can compare the data from the sensors after every 12 hours, so that we get a 
detailed description of the compared functioning of the car, thereby making it easy to judge if nay parts needs immediate attention or not. Finally all this data will be provided to the customer's either via a mobile application or a website under their personal accounts.

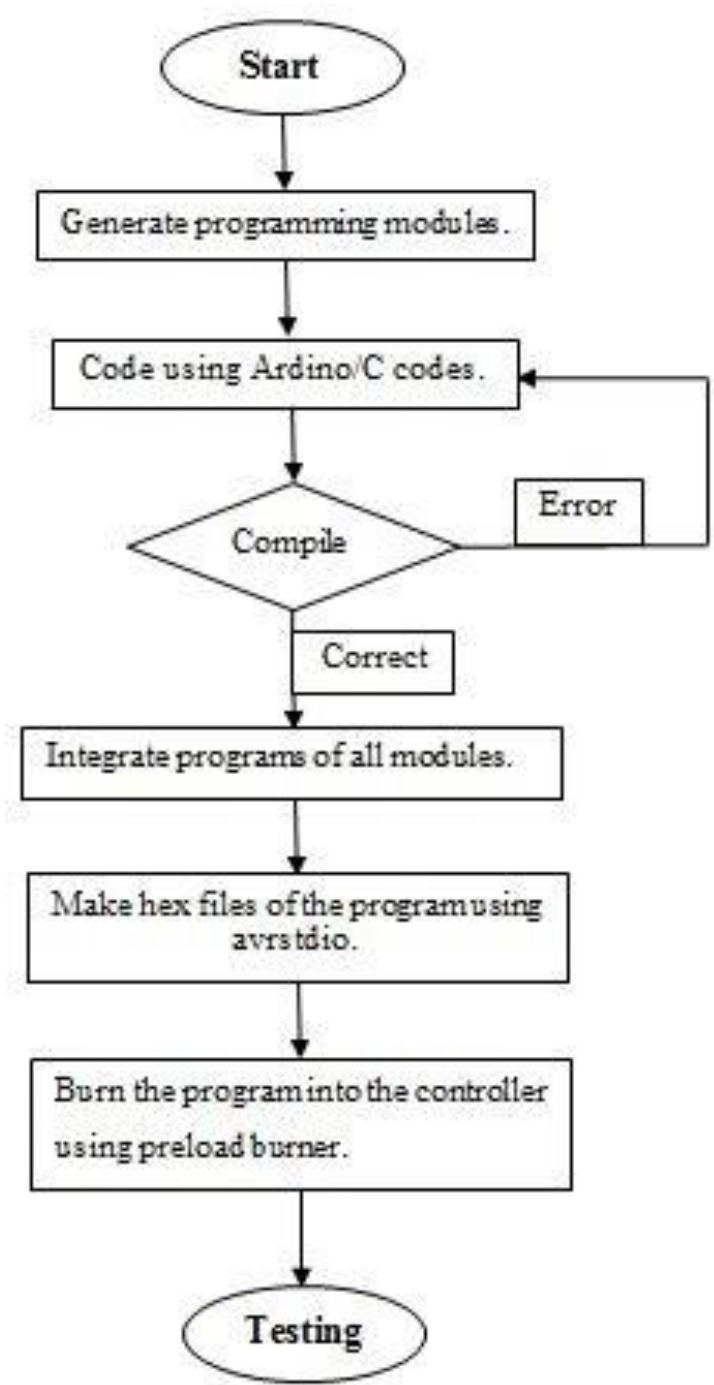

Fig. 6. Software Programming Flow Chart

\subsection{Hardware}

System Structure: The hardware system structure of the automotive health monitoring system consists of mainly a micro-controller board, power supply unit, GSM module, a buzzer, temperature sensor, hall effect flow rate sensor, a shock vibration sensor and an LCD-I2C module. The various sensors are installed in the functioning parts of the cars and then connected via GSM module to the cloud services. The system structure is shown in Figure 8.

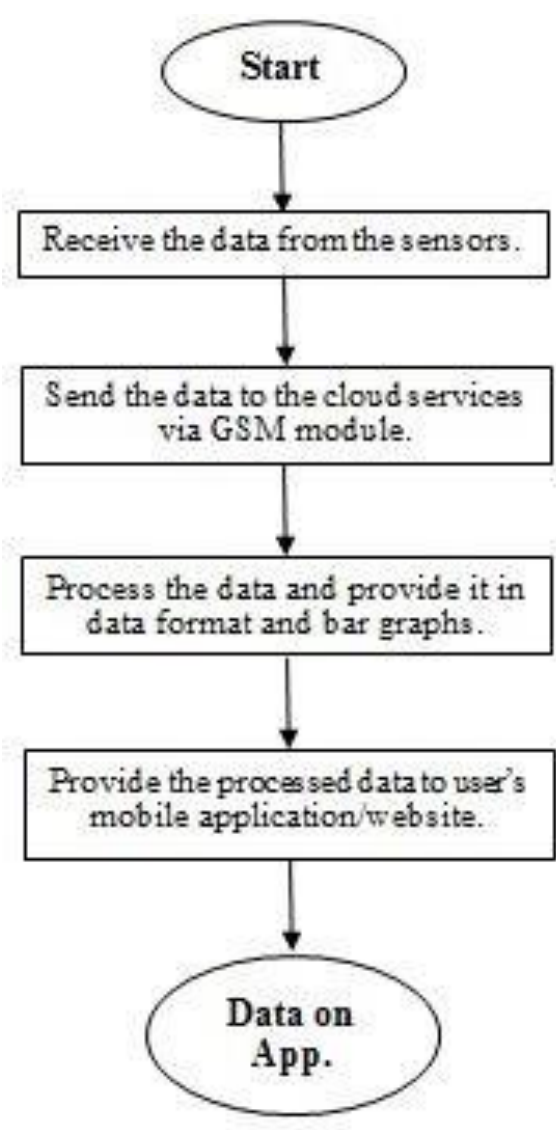

Fig. 7. Data Communication Flow Chart

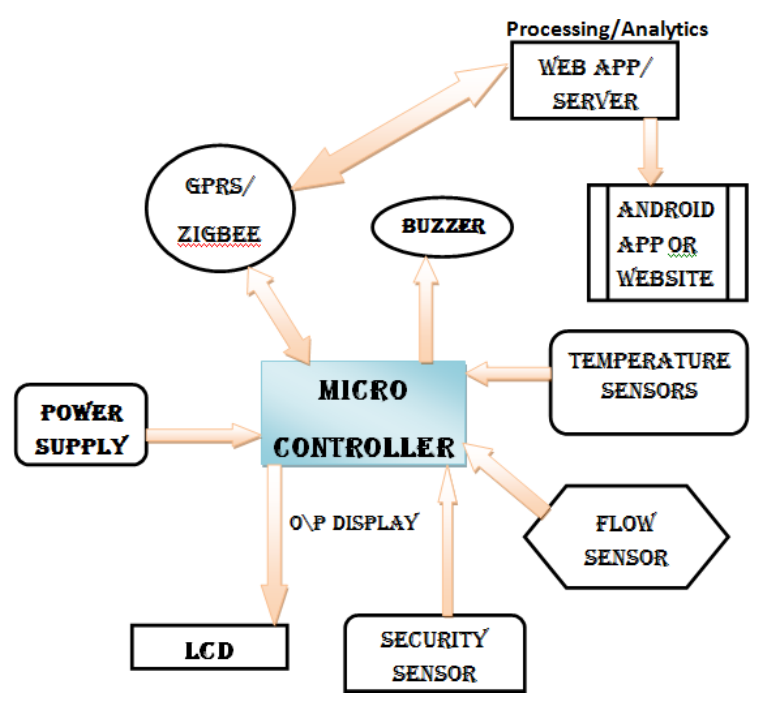

Fig. 8. System Structure

Hardware Process Flow: To understand the hardware process flow chart first and fore- most thing is to study and plan the circuit and components to be used. Then break the complete circuit in different modules and prepare block diagrams for each. Test the circuit for all individual units. Configure the complete circuit with the help of the block diagrams. Again simulate the complete circuit to test for any errors present or not, if any troubleshoot the errors and configure the final 
circuit. Design a PCB according to the circuit, and accordingly assemble the components on the $\mathrm{PCB}$ and with the help of soldering make the device stable and working. Test the device under various real time configurations to check for its effective working. Finally interface the whole device setup with the GSM to send the real time data to the client. Also connect the device with a buzzer/alarm to be installed with the device for emergency indications. Through all this interfacing, the microprocessor can have real time monitoring of the automotive, the pressure, temperature variations of the automotive will be known to the user thereby the security of the vehicle will be in the hands of the users.

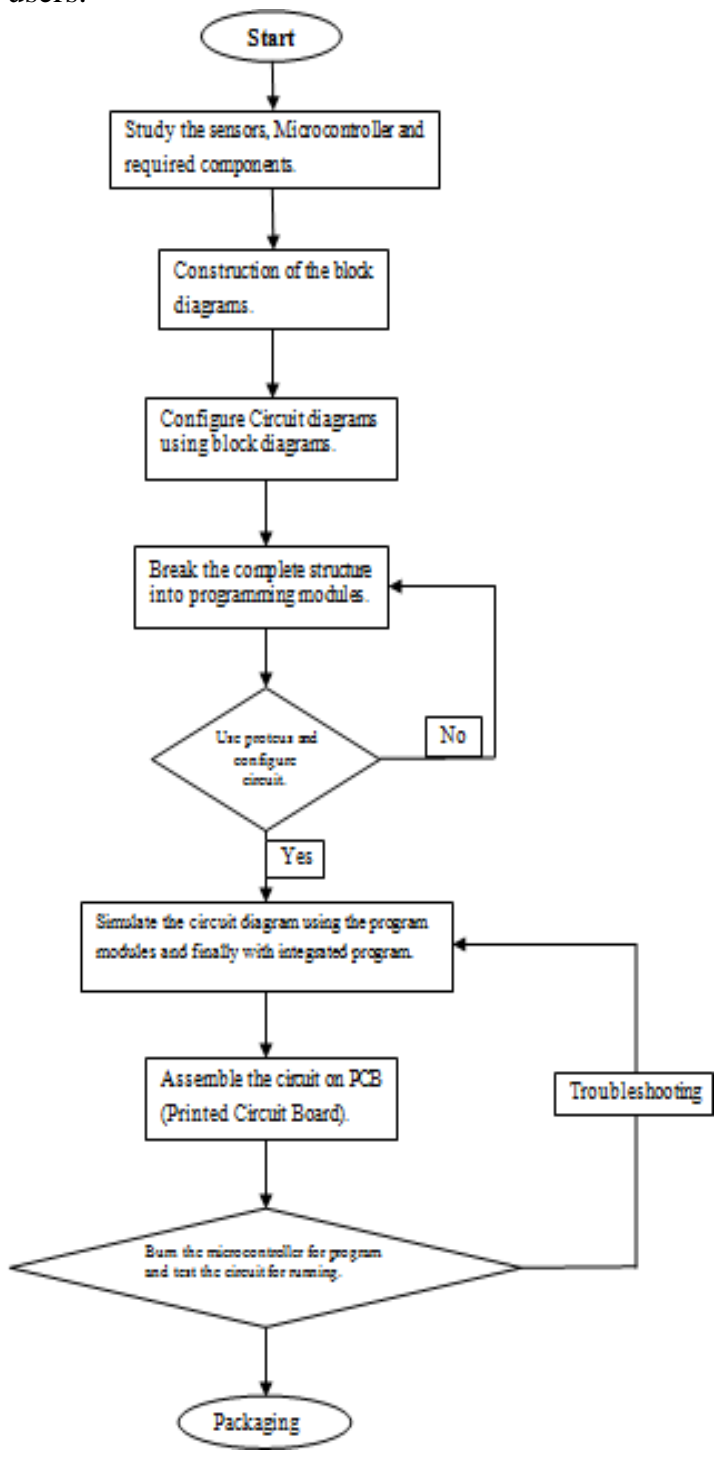

Fig. 9. Hardware Flow Chart

\section{COST BENEFITS}

Predictive maintenance is being protected that the main- tenance of vehicle is completed before the breakdown. It saving a cost and time period in terms of servicing and maintenance charges. Cost savings are 8 to 12 percent higher in predictive maintenance related to preventive maintenance. When we compare with reactive maintenance the cost savings vary from 30 to 40 percent. And the reduction in maintenance cost is 25 to 30 percent. 55 percent sudden failure is also reduced in predictive maintenance. Thus, the finance and labor cost is also reduce. There is an increase in production from 20 to 25 percent. When periodic maintenance assigns the ROI, the maintenance department produces a source of cost saving and excessive profit.

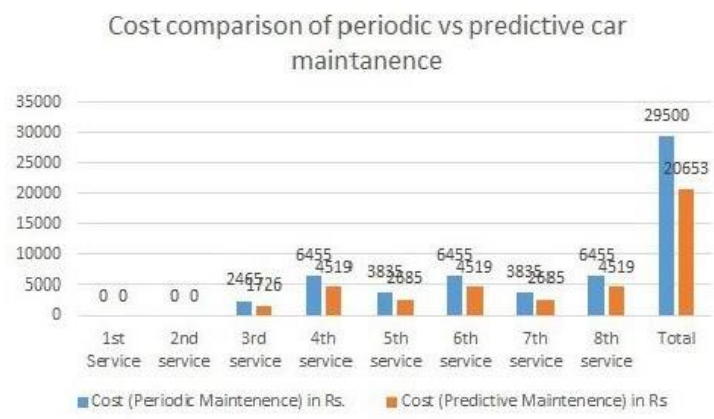

Fig. 10. Cost Comparison Bar Graph

\section{CHALLENGES}

There is a particular challenge in predictive maintenance is to setup data flow evenly from machines to application systems. It is beneficial for us to attain the higher security and reliability with the minor delay. An easy connection and communication with machines, devices, sensors and people is also needed. Skills and knowledge are also required. Selecting the right technique for the right problem and to know the output of the program much better. Whenever, we introduce new technology in market the biggest problem we face is the acknowledgement of the technology because people are count on their earlier technology and persuading them is also a challenge for us. There is a limitation in predictive analytical solutions to provide data regarding future tendency. They do not provide under-standing to end users which can help them to take action. Therefore, users will need to shift to another tool, which may interrupt their workflow.

\section{CONCLUSION}

The technology today seems to come up with a new concept every now-and-then, which seems to a grand challenge for the budding engineering community. The requirement of perform- ing a monitoring based on the tremendous economic and life- safety benefits this technology offers is the most alluring fact for any industry as it detects the damages of its structural and mechanical framework before it even encounters. This will not only detect the condition of the car but also highlight the 
parts that require maintenance and ensure safety of both car and its owner. The detail of the car will be provided in the efficient and effective manner with the help of data and bar graphs. The mobile application software will interact with the hardware interface unit wirelessly via Bluetooth to acquire desired vehicle parameters displayed on the LCD board. These readings will be displayed locally to the user. We used application to access the sensors information based on the GSM technology, thereby giving all the data to the concerned user. With the increasing trend of smart phones and wireless communication, it has become feasible to use these technologies for real time solutions. Despite limited resources these technologies are being used along with machine learning approaches to solve big problems in automotive industry.

To elevate this device with more technical features further enhancements can also be done in future. For-instance: to protect the vehicle from unauthorized users we can install acamera inside the car which will enable to have photos of rightful owner along with audio and video, with the photos being sent over the M2M link. We can also use a tracking device to track the current location of the vehicle if it gets to an unauthorized user or to an unauthorized location. The mobile application that we have done in synchronization with the web page can be further modified by including more advanced features with existing ones. Also as we see the vehicle technology is moving towards electric cars, we can also keep a research specific to the battery materials that charges in lesser time and has higher run duration. Therefore, a wide range of applications can be implemented to make this system a more desirable and beneficial as well.

\section{REFERENCES}

[1] Baffour-A, E., Service Quality in the Motor Vehicle Maintenance and Repair Industry: A Documentary Review. International Journal of Engineering and Modern technology (2018, Vol 4(1)).

[2] Dhall R. and Solanki V., An IoT Based Predictive Connected Car Maintenance. International Journal of Interactive Multimedia and Artificial Intelligence (2017 Vol 4(3)).

[3] KR, Parmesh and Neriya, Rashmi. Vehicle Health Monitoring and Analysis (2016).

[4] Wen, Z. and Meng, J., Design of vehicle positioning system based on ARM. In International Conference on Business Management and Electronic Information (2011,Vol. 4).

[5] Nwadiogbu E.O., Mylaraswamy D., Menon, S., Voges, H.C and Had- den, G., Honeywell International Inc, Vehicle health monitoring system architecture for diagnostics and prognostics disclosure (2013).

[6] Kumar, T.N. and Sivaji, S., Android-Based Vehicle Monitoring and Tracking System Using ARM7 and CAN Technology (2015) 